\title{
The Effect of Longitudinal Training on Working Memory Capacities: An Exploratory EEG Study
}

\author{
Zian Pei ${ }^{1}$, Tao $\mathrm{Xu}^{1}$, Anastasios Bezerianos ${ }^{2}$, Junhua $\mathrm{Li}^{1,3}$ Senior Member, IEEE, \\ Yu Sun ${ }^{4}$ Senior Member, IEEE and Hongtao Wang ${ }^{1,2, \dagger}$ Member, IEEE
}

\begin{abstract}
The study of working memory (WM) is a hot topic in recent years and accumulating literatures underlying the achievement and neural mechanism of WM. However, the effect of WM training on cognitive functions were rarely studied. In this study, nineteen healthy young subjects participated in a longitudinal design with one week $N$-back training $(N=1,2,3,4)$. Experimental results demonstrated that training procedure could help the subjects master more complex psychological tasks when comparing the pre-training performance with those post-training. More specifically, the behavior accuracy increased from $68.14 \pm 9.34 \%, 45.09 \pm 14.90 \%, 39.12 \pm 12.71 \%$, and $32.11 \pm 10.98 \%$ for 1-back, 2-back, 3-back and 4-back respectively to $73.52 \pm 4.01 \%, 69.14 \pm 5.28 \%, 69.09 \pm 6.41 \%$ and $\mathbf{6 4 . 4 1} \pm 5.12 \%$ after training. Furthermore, we applied electroencephalogram (EEG) power and functional connectivity to reveal the neural mechanisms of this beneficial effect and found that the EEG power of $\delta, \theta$ and $\alpha$ band located in the left temporal and occipital lobe increased significantly. Meanwhile, the functional connectivity strength also increased obviously in $\delta$ and $\theta$ band. In sum, we showed positive effect of WM training on psychological performance and explored the neural mechanisms. Our findings may have the implications for enhancing the performance of participants who are prone to cognitive.
\end{abstract}

\section{INTRODUCTION}

Working memory (WM) plays an important role in daily activities because it provides the basis for advanced cognitive functions. One of the most popular experimental paradigms in WM research is the n-back task [1] [2]. Much work has been made to elucidate the potential mechanism of brain during $\mathrm{n}$-back tasks using functional neuroimaging technology, such as functional magnetic resonance imaging (fMRI) and electroencephalogram (EEG). Among these techniques, EEG is widely used in research because of its advantages of easy accessibility and excellent temporal resolution.

A major topic in the study of WM has been on how the changes of frequency band power by WM tasks with

*This work is supported by Natural Science Foundation of Guangdong Province (2018A030313882), Projects for International Scientific and Technological Cooperation (2018A05056084), Science Foundation for Young Teachers of Wuyi University (2018td01), Jiangmen Brain-like Computation and Hybrid Intelligence R\&D Center ([2018]359, [2019]26) and Start up funds for scientific research of high-level talents of Wuyi University (5041700168).

1 Faculty of Intelligent Manufacturing, Wuyi University, Jiangmen, 529020, China.

2 Centre for Life Sciences, National University of Singapore, 117456, Singapore.

${ }^{3}$ School of Computer Science and Electronic Engineering, University of Essex, Colchester, CO4 3SQ, UK

${ }^{4}$ Department of Biomedical Engineering, Zhejiang University, Zhejiang 310027, China.

${ }^{\dagger}$ indicates the corresponding author (email: nushongtaowang@qq.com). different difficulty levels. A few studies have shown that frontal mid-line theta band increased in magnitude as WM load increased [3]. In contrast, posterior alpha band power decreased as WM load increased [4]. Moreover, Chen et al. found that posterior alpha power decreased, and temporal region-distributed beta power increased as WM load increased [5]. Imperatori et al. compared the EEG power spectra of five frequency bands $(\delta, \theta, \alpha, \beta$ and $\gamma)$ with different levels of difficulty (1-back vs. 3-back) [6]. According to the statements in the above studies, WM seems to be relevant to all typical frequency bands. Therefore, we included all typical frequency bands in this study. In addition to band power features, functional connectivity features were recently used in the WM analysis. The functional connectivity features can provide inter-channel information representing interactions between brain regions, which cannot be captured by power features that are derived from individual channels. Jlenia et al. explored the topology of the relevant EEG-derived brain networks during WM tasks [7].

In recent years, scientists began to focus on WM training. Green et al. proposed possible characteristics of training programs that may help enhance learning [8]. Martin et al. evaluated the effectiveness of cognitive training in adulthood and old age [9]. Others studied the changes of functional brain network after cognitive training. For example, Hempel et al. [10] observed the effect of visual spatial work training on brain activity. Buschkuehl et al. [11] found that there were differences in brain activity between 4-back and 1-back. As a system for short term maintenance and manipulation of task-relevant information [12], WM is inherently involved in all higher-level cognitive activities. Accordingly, if WM training is successful, it may have a broad impact on individuals. However, subsequent training studies showed that there was no consistent conclusion [13] [14] [15] [16]. To solve this problem, we explore the changes of sub-band in EEG power and functional connectivity strength before and after WM training in a longitudinal study design.

\section{MATERIALS AND METHODOLOGY}

\section{A. Participants}

Nineteen healthy young people (age: $22.4 \pm 2.6$ years) participated in this study. All subjects were right-handed and had normal or corrected-to-normal vision. They were prohibited of taking any psychiatric and/or neurologic drugs in the 1 week before the study. The protocol of the experiment was approved by the institutional review board of the National 
University of Singapore. All participants signed the informed consent form before starting the experiment.

\section{B. Experimental paradigms}

The subject was asked to complete two sessions within one week and did training every other day, thus each subject needed to train twice. In each session, different n-back tasks appeared randomly, the subject needed to make the choice according to the target by pressing the "space". This behavior was recorded and be denoted as an experimental evaluation index (behavior accuracy). There were 240 trails total in each session. During the experiment, 62-channel $\mathrm{Ag} / \mathrm{AgCl}$ electrodes were employed for EEG recording with a sampling rate of $256 \mathrm{~Hz}$ and band-pass filter $(0.5 \sim 70 \mathrm{~Hz})$ was used for signal filtering.

\section{Data Processing}

The trails of responses (correct or incorrect) during nback task were recorded and used for further analysis. A typical procedure was utilized to mitigate artifacts from EEG signals, including bandpass filter $(0.5 \sim 48 \mathrm{~Hz})$ and independent component analysis (ICA) [17]. Each epochs contained a time window of $3000 \mathrm{~ms}$, which was extracted from $-500 \mathrm{~ms}$ to $2500 \mathrm{~ms}$ according to the target onset. The time period of $-500 \mathrm{~ms}$ to $0 \mathrm{~ms}$ was used as the baseline. Each epoch will be used for the EEG power features and functional connectivity features extraction.

\section{Methods}

1) Electroencephalogram Power: Fourier transform (FT) was used to obtain power features in five typical EEG frequency bands $(\delta: 1 \sim 4 \mathrm{~Hz}, \theta: 4 \sim 8 \mathrm{~Hz}, \alpha: 8 \sim 12$ $H z, \beta: 12 \sim 30 H z$, and $\gamma: 30 \sim 45 H z)$. Specifically, for each EEG data epoch, the power of each EEG channel in five sub-bands were obtained by using FT. The channels were then segmented into five areas: frontal location, left temporal location, right temporal location, parietal location and occipital location. There were five sub-band power features in each frequency band by averaging the values of the power within each region. Finally, EEG power increment after training is calculated for analysis, which is obtained by the EEG power of each sub-band in each region after training subtracts the values of before training.

2) Functional Connectivity: Interactions between brain regions could be quantified by Phase Lag Index (PLI). PLI solves problems caused by volume conduction, common source and active reference in traditional methods (such as Phase Locking Value and Partial Directed Coherence). The PLI is computed with the following formula:

$$
P L I=\left|\left\langle\operatorname{sign}\left[\sin \left(\varphi_{i}-\varphi_{k}\right)\right]\right\rangle\right|
$$

where sign stands for signum function, || indicates absolute value function and \langle\rangle stands for arithmetic average operation. $\varphi_{i}, \varphi_{k}$ are the phases of EEG channels $i$ and $k$, obtained by the Hilbert transformation. In our case, a connectivity matrix with the size of $62 \times 62$ was obtained by
PLI for each band. We got one value of PLI in each band by averaging the values in the connectivity matrix. Finally, the value obtained by the connectivity strength in sub-band after training subtracts the strength of before training is calculated for further analysis.

\section{RESULTS}

\section{A. Behavior Results}

In Table I, we provided the averaged behavior accuracy across nineteen subjects during N-back experiments $(\mathrm{N}=1,2,3,4)$. It can be observed that in both session 1 and session 2 , the behavior accuracy decreased with the increase of task difficulty (from 1-back to 4-back). The highest accuracy obtained in 1-back, which was $68.14 \pm 9.34 \%$ and $73.52 \pm 4.01 \%$ in session 1 and session 2, respectively. Interestingly, the WM training indeed can help to enhance the performance of the subject. The more complex of the task, the more significant effect could be obtained. Furthermore, the standard deviation of session 2 was much smaller than that of session 1, which indicates that the performance of subject is more stable after training procedure.

TABLE I: The averaged behavior accuracy across nineteen subjects of WM task (N-back) in before and after training sessions.

\begin{tabular}{ccccc}
\hline \multirow{2}{*}{ Sessions } & \multicolumn{4}{c}{ Accuracy (\%) $($ Mean \pm std) } \\
\cline { 2 - 5 } & 1-back & 2-back & 3-back & 4-back \\
\hline Before & $68.14 \pm 9.34$ & $45.09 \pm 14.90$ & $39.12 \pm 12.71$ & $32.11 \pm 10.98$ \\
After & $73.52 \pm 4.01$ & $69.14 \pm 5.28$ & $69.09 \pm 6.41$ & $64.41 \pm 5.12$ \\
\hline
\end{tabular}

\section{B. Electrophysiological Results}

As shown in Fig.1, it provides the averaged power changes across all subjects in each sub-band located in different brain region.

Delta band: The changes of EEG power in occipital were significant. The power of all tasks (n-back) increased in left temporal, parietal and occipital. In frontal, the power had no changes in 1-back decreased in 4-back, and increased in the other two tasks. In parietal, the power of all tasks increased, except for 4-back. In all tasks, the power increased in 2-back were higher compared with other tasks and only 4-back for power decreased in frontal and right temporal. In addition, the power in parietal and occipital in 3-back were higher than other three brain region in 3-back.

Theta band: The power decreased during 1-back in frontal and right temporal, and the power increased under all other conditions. Moreover, the reduced power in right temporal was more than that in frontal. In all cases of increased power, power increment is the largest for each task in left temporal, and the minimum increased in power was in the right temporal.

Alpha band: Power reduction only occurs in right temporal. In right temporal, power decreased in 1-back and 4back and increased in other tasks. In all cases of increased power, the lowest increment of power was obtained during 

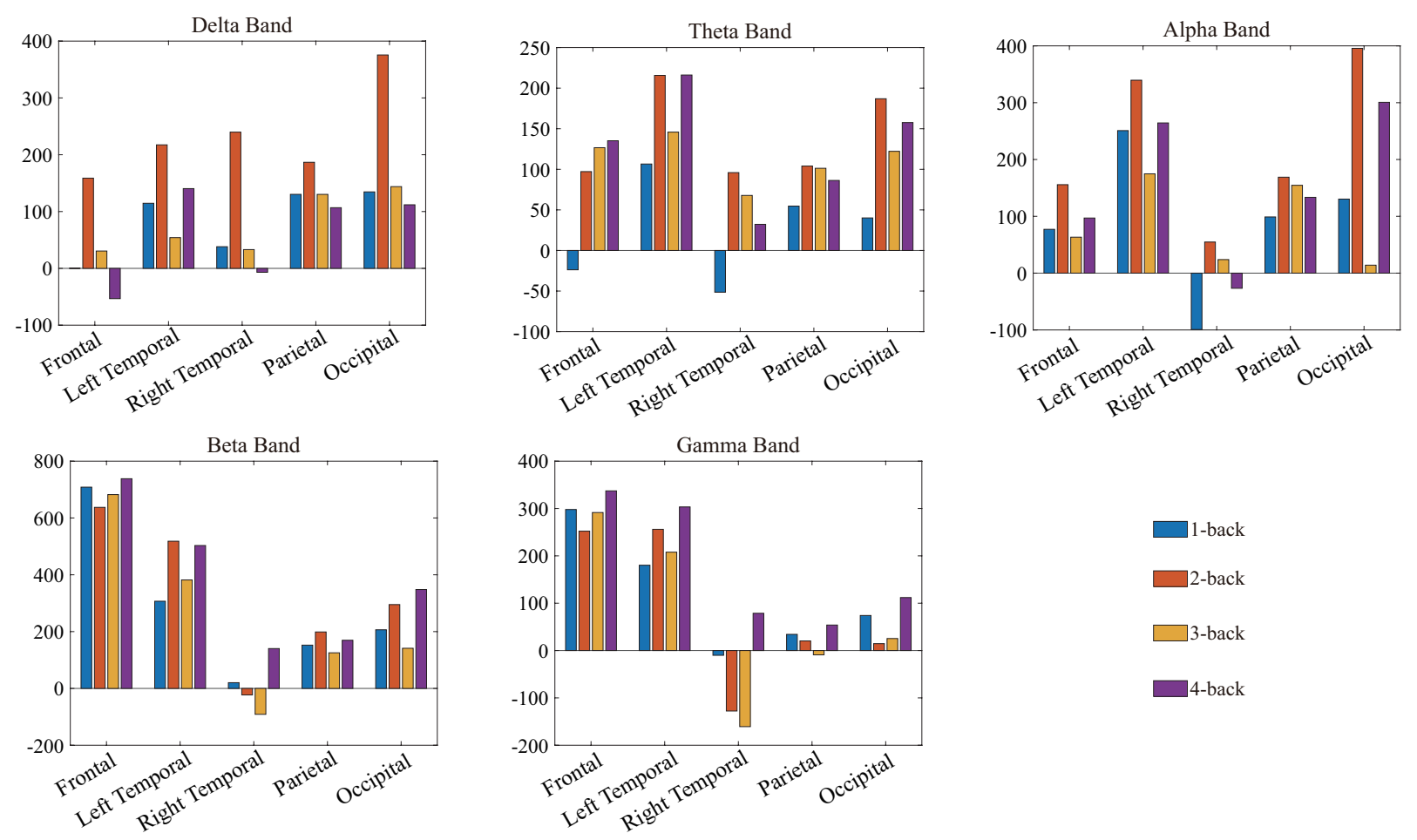

Fig. 1: The power changes averaged across all subjects in each sub-band and each brain region. The Y-axis stands for deference of power after training. The power increased significantly of left temporal and occipital among $\delta, \theta$ and $\alpha$ band, and the power increased significantly of frontal and left temporal between $\beta$ and $\gamma$.

3-back in occipital, and the highest increment of power was obtained during 2-back in occipital. From every brain region, the lowest increment of power was obtained in right temporal and the highest was obtained in left temporal.

Beta band: It is be seen that there is a significant increase of power in the frontal location compare with other brain regions. The power decreased in right temporal during 2back and 3-back and increased in all other conditions. From a numerical points of view, the increment of power in beta band was highest compared to other bands.

Gamma band: The main increments of power were in frontal and left temporal. In right temporal, the power decreased in all tasks besides 4-back task. In parietal, the power increased in all tasks besides 3-back task. In addition, the increment of power in parietal and occipital were lower compared with other bands.

The change of functional connectivity strength of each sub-band after training are shown in Fig.2. The results showed that the functional connectivity increased in $\delta$ and $\theta$ band during all tasks and the former was larger than the latter, while in $\beta$ band there is a decreased pattern. In addition, we found that connectivity strength began to decrease in $\alpha$ band.

\section{DISCUSSION}

In this study, we explored the changes of power in each band before and after training. The results show that the power increased during most tasks in each band after training. Specifically, in $\delta$ and $\theta$ band, the power enhancement is mainly in left temporal and occipital. Such a result indicates

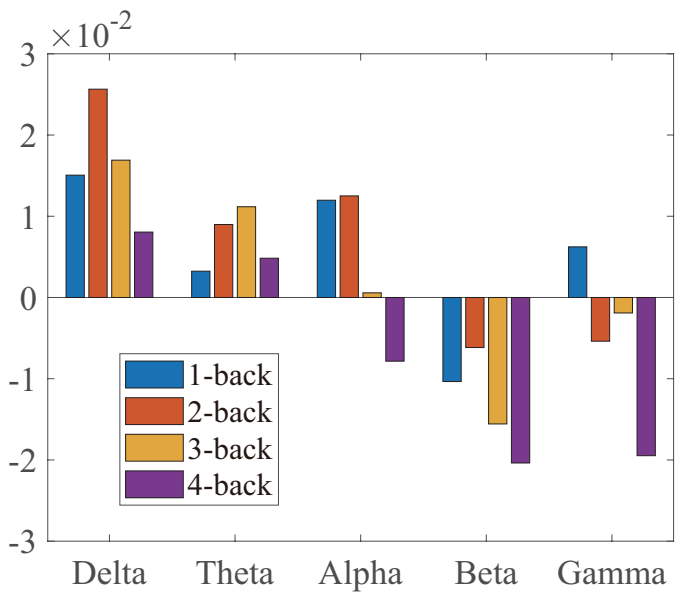

Fig. 2: The change of connectivity strength of sub-band after training. The Y-axis stands for difference of connectivity strength after training. The connectivity strength increased in $\delta$ and $\theta$ band of all tasks, and decreased in $\beta$ band.

that the power changes in $\delta$ and $\theta$ bands may be related to WM. It has been reported that significant changes occurred in the delta band during WM task [6], and continuous cognitive processing may be manifested as long-lasting increase of $\theta$ power [18] [19]. In $\alpha$ band, the power increased in most case especially in left temporal and occipital. It has been found that the power changes in $\alpha$ band seem to be related to memory performance [20] [21]. There is notable $\alpha$ band 
power increase when a subject does not pay attention to or focus on something [22]. This mannifests that the subjects do not need to mobilize a lot of attention in WM tasks after training. In $\beta$ and $\gamma$ band, the increment of power is mainly shown in frontal and left temporal. In addition, the increment of power in $\beta$ band is highest compared with other bands. It suggests the changes of $\beta$ power are associated with cognitive processes [23] [24].

Summing up these rules, we find that the main area of EEG power increased were left temporal and occipital among $\delta, \theta$ and $\alpha$ band, while in $\beta$ and $\gamma$ band, the main area of EEG power increased were frontal and left temporal. The left temporal seems to play an important role in WM training. Imperatori et.al found that the modification caused by the increased complexity of tasks are most significant in the left temporal lobe structures [6]. The researchers also found the node showing the greatest connectivity values was located in the left middle temporal region [24]. In addition, the changes of power in left temporal were different from that in right temporal. Such a result shows the hemispheric asymmetry of the left and right brain.

We also explored the changes of connectivity strength before and after training. The result shows that significant changes were obtained in $\delta$ and $\beta$ band. The connectivity strength of delta band increased, while connectivity strength of $\beta$ band decreased. With the increase of frequency, the strength of connection changes from increase to decrease.

This study explored the WM training in terms of EEG power and functional connectivity strength. However, there are still some limitations, which should be paid attention in future studies. Firstly, in this study, we did not discuss the connection patterns of different regions of the brain. Secondly, this study only considers the change of connectivity strength, and does not involve the topological analysis of the network. Existing studies have proven that network topology has obvious changes in WM training. Langer et al. [25] found that an increase in the small-worldness with in a distributed fronto-parietal network.

\section{CONCLUSION}

In the present study, we designed an experiment of WM based on n-back tasks (1-back, 2-back, 3-back and 4-back), as a comparison experiments each subject completed two sessions. Experimental results proved that the training procedure could help to enhance the performance and lead to a higher accuracy. Moreover, to compare the changes between before and after training, two methods of EEG power and functional connectivity strength were employed. We found that the EEG power increased in all sub-band and the left temporal seemed to play an important role in WM training, which presented the hemispheric asymmetry. In addition, the changes of functional connectivity strength increased in $\delta$ and $\theta$ band and decreased in $\beta$ band. This phenomenon manifests that the training procedure have a significant impact on WM. In the future, we will recruitment the elderly group and increase training times to four sessions for comprehensive analysis.

\section{REFERENCES}

[1] Conway, Andrew RA, et al. "Working memory span tasks: A methodological review and user's guide." Psychonomic bulletin \& review 12.5 (2005): 769-786.

[2] Ragland, J. Daniel, et al. "Working memory for complex figures: an fMRI comparison of letter and fractal n-back tasks." Neuropsychology 16.3 (2002): 370-379.

[3] Gevins, Alan, et al. "High-resolution EEG mapping of cortical activation related to working memory: effects of task difficulty, type of processing, and practice." Cerebral cortex (New York, NY: 1991) 7.4 (1997): 374-385.

[4] Lei, Shengguang, and Matthias Roetting. "Influence of task combination on EEG spectrum modulation for driver workload estimation." Human factors 53.2 (2011): 168-179.

[5] Chen, Youguo, and Xiting Huang. "Modulation of alpha and beta oscillations during an n-back task with varying temporal memory load." Frontiers in psychology 6 (2016): 2031.

[6] Imperatori, Claudio, et al. "Modifications of EEG power spectra in mesial temporal lobe during n-back tasks of increasing difficulty. A sLORETA study." Frontiers in human neuroscience 7 (2013): 109.

[7] Jlenia Toppi, et al. "Different Topological Properties of EEG-Derived Networks Describe Working Memory Phases as Revealed by Graph Theoretical Analysis.” Frontiers in Human Neuroscience 11 (2018): 637-641.

[8] Green, C. Shawn, and Daphne Bavelier. "Exercising your brain: a review of human brain plasticity and training-induced learning." Psychology and aging 23.4 (2008): 692-701.

[9] Lödén, Martin, et al. "A theoretical framework for the study of adult cognitive plasticity." Psychological bulletin 136.4 (2010): 659-676.

[10] Hempel, Albrecht, et al. "Plasticity of cortical activation related to working memory during training." American Journal of Psychiatry 161.4 (2004): 745-747.

[11] Buschkuehl, Martin, et al. "Neural effects of short-term training on working memory." Cognitive, Affective, \& Behavioral Neuroscience 14.1 (2014): 147-160.

[12] Baddeley, Alan. "The episodic buffer: a new component of working memory?." Trends in cognitive sciences 4.11 (2000): 417-423.

[13] Bürki, Céline N., et al. "Individual differences in cognitive plasticity: an investigation of training curves in younger and older adults." Psychological Research 78.6 (2014): 821-835.

[14] Waris, Otto, Anna Soveri, and Matti Laine. "Transfer after working memory updating training." PloS one 10.9 (2015): e0138734.

[15] Thompson, Todd W., et al. "Failure of working memory training to enhance cognition or intelligence." PloS one 8.5 (2013): e63614.

[16] Redick, Thomas S., et al. "No evidence of intelligence improvement after working memory training: a randomized, placebo-controlled study." Journal of Experimental Psychology: General 142.2 (2013): 359-379.

[17] Chuang, Chun-Hsiang, et al. "An EEG-based perceptual function integration network for application to drowsy driving." KnowledgeBased Systems 80 (2015): 143-152.

[18] Philip Tseng, et al. "The critical role of phase difference in theta oscillation between bilateral parietal cortices for visuospatial working memory." Scientific Reports 8.1 (2018): 2071-2078.

[19] Pesonen, Mirka, et al. "Brain oscillatory 1-30 Hz EEG ERD/ERS responses during the different stages of an auditory memory search task." Neuroscience letters 399.1-2 (2006): 45-50.

[20] Krause, Christina M., et al. "The effects of memory load on eventrelated EEG desynchronization and synchronization." Clinical neurophysiology 111.11 (2000): 2071-2078.

[21] Pesonen, Mirka, et al. "Brain oscillatory 4-30 Hz responses during a visual n-back memory task with varying memory load." Brain research 1138 (2007): 171-177.

[22] Gloor, Pierre. "Hans Berger and the discovery of the electroencephalogram." Electroencephalography and clinical neurophysiology 28 (1969): 1-36.

[23] André M. Bastos, et al. "Laminar recordings in frontal cortex suggest distinct layers for maintenance and control of working memory." Proceedings of the National Academy of Sciences of the United States of America 115.5 (2018): 1117-1122.

[24] Yeo, BT Thomas, et al. "Functional specialization and flexibility in human association cortex." Cerebral cortex 25.10 (2015): 3654-3672.

[25] Langer, Nicolas, et al. "The effects of working memory training on functional brain network efficiency." Cortex 49.9 (2013): 2424-2438. 